\title{
Tourisme et Innovations : entre adaptations et
} (R)évolutions (1/2)

Tourism and Innovation: adaptation or revolution (1/2)

Aude Ducroquet et Philippe Viallon

\section{OpenEdition}

\section{Journals}

\section{Édition électronique}

URL : http://journals.openedition.org/tourisme/1887

DOI : 10.4000/tourisme.1887

ISSN : 2492-7503

Éditeur

Éditions touristiques européennes

\section{Référence électronique}

Aude Ducroquet et Philippe Viallon, «Tourisme et Innovations : entre adaptations et

(R)évolutions (1/2) », Mondes du Tourisme [En ligne], 13 | 2017, mis en ligne le 30 décembre 2017, consulté le 23 septembre 2020. URL : http://journals.openedition.org/tourisme/1887 ; DOI : https:// doi.org/10.4000/tourisme.1887

Ce document a été généré automatiquement le 23 septembre 2020.

\section{cc)}

Mondes du tourisme est mis à disposition selon les termes de la licence Creative Commons Attribution - Pas d'Utilisation Commerciale - Pas de Modification 4.0 International. 


\section{Tourisme et Innovations : entre adaptations et (R)évolutions $(1 / 2)$}

Tourism and Innovation: adaptation or revolution (1/2)

Aude Ducroquet et Philippe Viallon

\section{De l'invention à l'innovation}

Dans son ouvrage L'invention du tourisme, l'historien Marc Boyer (1996) rappelle que le statut de pratique sociale du tourisme ne l'empêche pas d'être une invention. Ses inventeurs, car ils sont plusieurs, sont les lords anglais qui, dépossédés du pouvoir politique, font et font faire à leur fils the tour sur le continent européen, voyage qui peut les mener aussi bien dans les "monts horribles $»^{1}$ que dans les villes de bains, voire jusqu'aux berceaux de la civilisation occidentale, Rome et Athènes. Ce tour a pour objet de découvrir par soi-même les valeurs de la civilisation et de contribuer à l'éducation des individus qui le pratiquent. C'est un bel objectif pour le tourisme, qui en tire son nom, mais qui pourrait encore aujourd'hui être questionné. Dans ses autres livres (Boyer et Viallon, 1994; Boyer, 2000), l'auteur montre que le tourisme s'est développé par imitation, d'une couche sociale à une autre, pour finalement concerner une grande partie de la population dans le courant du XXe siècle. Le fait d'être copié enlevant aux précurseurs le caractère distinctif de leur démarche (Veblen, 1979; Bourdieu, 1979), ceux-ci partent souvent inventer d'autres lieux. Aujourd'hui encore, le tourisme continue d'être inventé, même si les leaders d'opinion sont plus souvent américains qu'anglais et plus souvent issus du monde du show-business que de l'aristocratie. Cette invention peut prendre différentes formes :

- Des lieux sont inventés. De même que la famille Gould a «inventé » Juan-les-Pins dans les années 1920 ou Brigitte Bardot Saint-Tropez, un non-lieu peut devenir touristique : soit parce que sa caractéristique négative majeure devient, d'un coup, positive (exemple de la Vallée de la mort ou du tourisme industriel), soit parce qu'on crée à partir de rien un lieu attractif (cas des Centerparks). Cette touristicité (Botti, 2011) est donc bien la résultante à la fois de différentes caractéristiques du lieu, mais aussi de l'activité ou de la non-activité de 
certaines parties prenantes. Cela amène à se poser la question de la qualité du lieu (Coëffé et Violier, 2008).

- Des pratiques sont inventées. La deuxième moitié du XX⿳亠丷厂犬 ${ }^{\mathrm{e}}$ siècle abonde en exemples d'activités sportives ou de loisirs inventées, le plus souvent en Californie, et importées dans le reste du monde. Les sports de glisse en sont un bel exemple. Ils apportent leur lot de lieux choisis (les spots de surf), de nouveaux matériels, de nouveaux champions. L'ensemble des nouvelles pratiques proposées n'ont pas le même succès, loin s'en faut : le monoski ou le télémark, des variantes du ski, n'ont pas fait long feu, à l'inverse du snow-board qui a trouvé sa place parmi les pratiques de loisirs.

- Des modes de vie sont inventés. Ces nouveaux modes de vie sont le résultat des deux premières catégories d'inventions. Ils sont largement suggérés par des entreprises productrices de vêtements, de mobilier et d'accessoires divers, qui viennent chercher dans les pratiques touristiques des thèmes porteurs originaux.

2 Le lien entre invention et innovation est fort, mais les deux concepts ne sont pas synonymes: une innovation peut se construire à partir d'une invention, mais une invention ne donne pas forcément lieu à une innovation. L'invention du GPS, système de repérage par satellite, a d'abord eu un usage militaire, puis elle a permis des innovations dans le secteur du tourisme en permettant des excursions autonomes, dans lesquelles le touriste est guidé grâce à son smartphone qui dispose de cette fonctionnalité et grâce à l'application qu'il a téléchargée. En revanche, lorsque Papin a inventé l'autocuiseur au XVII ${ }^{\mathrm{e}}$ siècle, cela n'a dans un premier temps donné lieu à aucune innovation.

\section{Les innovations dans le tourisme, un traitement marketing de la pratique}

3 Depuis que le tourisme est devenu un produit industriel à la fois par le nombre d'individus concernés - le milliard de touristes internationaux a été dépassé en $2012^{2}-$ et par le chiffre d'affaires du secteur - plus de 1100 milliards d'euros en $2016^{3}$-, c'est plus que jamais le marketing qui préside à sa destinée. La professionnalisation du secteur est très forte, les secteurs publics et privés sont fortement impliqués dans sa promotion et la majorité des hommes politiques voit dans le tourisme une solution à de nombreux problèmes économiques et sociaux. Parmi toutes les qualités que l'on peut attribuer à un produit ou à un service, l'une des plus évidentes est la nouveauté. L'affirmation de l'innovation fait donc partie de la rhétorique du marketing : dans un marché souvent saturé, comment faire la différence par rapport à l'existant si ce n'est en revendiquant la nouveauté ? Dans une société où la technologie prend une place croissante et évolue très vite, la contrainte sociale de se tenir à la page est forte. La nouveauté semble être l'un des moteurs essentiels de l'économie. Y aurait-il une obsolescence des pratiques touristiques comme il y a une obsolescence des appareils techniques? On a bien vu, par exemple, que certaines stations thermales ont su prendre le virage du bien être tandis que d'autres périclitaient (Lohez, 2000).

4 La nouveauté, dans le tourisme, peut être revendiquée grâce à plusieurs éléments :

- La technologie. Celle-ci peut être à l'origine de l'innovation, comme dans l'exemple du GPS mentionné plus haut, ou la faciliter: si l'hébergement chez l'habitant existe depuis longtemps, Internet lui a donné une dimension inédite (Airbnb). Cela peut être aussi un plus 
de technologie : grâce à la technologie, un manège d'un parc de loisirs sera encore plus rapide que le précédent.

- L'espace concerné. Les innovations mondiales sont rares. Plus fréquentes sont les imitations nationales, régionales ou locales, parfois légèrement modifiées pour éviter les problèmes juridiques - même si on ne peut pas protéger une pratique - ou pour s'adapter à la culture locale. L'exemple du surf « importé » en Aquitaine est bien décrit par Guibert (2011).

- La configuration de l'offre. Le camping fait la joie des amoureux de la nature depuis longtemps, mais il suffit de remplacer la tente traditionnelle par un tipi, pour offrir une nouveauté.

- Lorsque qu'un prestataire offre pour la première fois un service, il peut se targuer de nouveauté, même si la concurrence a déjà investi le domaine.

- Il y a également nouveauté lorsqu'une pratique traditionnelle est transformée en événement avec un retentissement dans les médias, comme dans le sport par exemple (Bessy et Suchet, 2015).

- Le sentiment de nouveauté tient enfin au touriste lui-même. Même si le rafting existe en France depuis les années 1980, un touriste le pratiquant de nos jours pour la première fois ressent son activité comme une nouveauté.

5 On comprend dès lors que l'innovation touristique est polymorphe et il est délicat de lui apporter une définition synthétique, au risque d'être trop général ou d'exclure une partie des changements ou des nouveautés qui apparaissent. Cette difficulté s'illustre dans les différentes propositions d'articles qui ont été faites pour ce numéro. Les articles proviennent de disciplines différentes (sociologie, sciences de l'information et de la communication, géographie, ressources humaines, économie, marketing...) et abordent la question de l'innovation sous des angles multiples mais complémentaires. Mondes du tourisme est depuis ses origines une revue inter- et transdisciplinaire et elle le montre encore une fois dans ce numéro.

6 Le rapport de la direction générale des entreprises (DGE) sur l'innovation touristique (2016) dresse un portrait de l'innovation dans le secteur du tourisme : l'innovation y serait une innovation digitale $(80 \%)$ à destination de l'usager final $(80 \%)$ et orientée vers les loisirs ( $90 \%)$. Dans ce secteur, souvent considéré comme low tech, ce sont, en grande partie, les technologies qui apportent la nouveauté, directement ou indirectement, et qui révolutionnent la manière de voyager et le rapport au voyage.

\section{Quatre exemples d'innovation}

7 Ainsi Pauline Neveu revient-elle sur le développement du couchsurfing. Cette pratique n'est en rien nouvelle, car l'accueil de touristes (notamment de pèlerins) par l'habitant existe depuis la nuit des temps. Néanmoins, elle s'est structurée et démocratisée grâce à Internet, qui a permis de mettre en relation plus facilement les demandeurs et les offreurs de logements. L'innovation technologique (Internet et les réseaux sociaux) a facilité l'apparition d'un nouveau type d'hébergement (innovation de prestation), s'inscrivant dans l'économie collaborative (innovation de business models différents de l'économie traditionnelle) et dans une nouvelle envie des touristes de s'immerger au sein d'un univers spécifique (évolution des modes de vie, innovation sociale et sociétale). Pourtant, Pauline Neveu note que l'innovation réside peut-être ailleurs. Sa vision de sociologue permet de mettre en évidence les rapports qui se nouent entre ces inconnus qui se rencontrent. Contrairement à ce qu'on aurait pu penser, cela permet de 
rassurer le touriste, qui se retrouve finalement dans un espace relativement sécurisé, un lieu d'équilibre, proche du familier.

8 L'expérience de visite est souvent au cœur des innovations. Falk et Dierking (2000) mettent au centre des conditions de cette expérience les contextes à la fois social, physique et personnel. Dans son étude d'une expérience de visite scolaire au musée mémorial d'Auschwitz-Birkenau, Nathanael Wadbled explique le parti pris de certaines structures, qui choisissent de se positionner sur une dimension particulière, comme par exemple: l'éducation identitaire, l'apprentissage de l'histoire ou encore le simple plaisir récréatif. Il démontre que la combinaison de ces dimensions permet d'apporter une expérience spécifique, différente de ce que l'on trouve dans une majorité des sites historiques. Ainsi, l'innovation des musées mémoriaux résiderait dans l'association de ces dimensions, en abandonnant le clivage entre les différents types de tourisme: de mémoire, culturel et récréatif. Même si l'on se situe, dans le cas des voyages scolaires traité dans cet article, à la limite des pratiques touristiques, cette approche permet de mettre le doigt sur un moteur commun aux voyages scolaires et au tourisme, qui est le fait d'approcher les réalités du monde.

Les territoires ne sont pas en reste et eux aussi se montrent innovants. Que ce soit dans leurs structures (évolutions des offices de tourisme), dans leur communication et leur marketing ou dans leur développement. C'est ce que montrent Fabrice Caudron et Céline Thomas, à travers une étude de cas franco-belge. La mise en place d'une coopération entre deux territoires transfrontaliers a permis de créer un nouvel espace touristique. En partant de la volonté de créer un circuit de randonnée familial (InnoRando pour tous) et donc de développer en commun des innovations de produits touristiques, les collectivités associées ont contribué à l'émergence d'un territoire touristique. Les auteurs soulignent que ces innovations sont directement liées au développement des nouveaux outils web et mobile. Au terme de cette étude, les auteurs posent la question de l'évolution d'un territoire et de sa perception par les acteurs touristiques via la création d'une nouvelle « personnalité » pour la destination.

10 L'ensemble de ces changements provoque indéniablement des renouveaux organisationnels. L'autonomisation des touristes, leurs besoins toujours plus importants de personnalisation entraînent les gares et les aéroports à proposer de nouveaux services et à concevoir autrement leurs halls et lieux d'attente. De même, les offices de tourisme et les agences cherchent à accueillir différemment le client et à repenser leur métier. Ainsi, voit-on apparaître des animateurs numériques de territoire, des metteurs en scène de l'offre touristique, des analystes de données, des promoteurs de marketing territorial... L'article que propose Camille Bernetière s'inscrit pleinement dans cette réflexion. En se basant sur deux cas (les villes de New York et de Mulhouse), elle présente deux stratégies managériales d'adaptation à la demande de clients différents. L'une s'appuie sur l'autonomisation du visiteur et transforme le conseiller en séjour en médiateur numérique. L'autre cherche à répondre aux besoins de personnalisation de l'usager en développant pour lui un rôle d'ambassadeur du territoire.

11 Ces quatre articles apportent des exemples, des éléments de réflexion, des questionnements sur des innovations de natures différentes : innovations en termes de produits, de destinations, de modèles économiques, d'usages, de métiers, de services, innovation technologique, sociale et sociétale, organisationnelle... Mais ils montrent surtout qu'une innovation n'intervient jamais seule et que c'est souvent la combinaison 
de plusieurs innovations partielles qui construisent l'innovation principale. L'innovation entraîne donc l'innovation et elle est, comme le soulignent Salvador et Jolibert (2017), un défi. Ce défi concerne également la recherche, qui est de plus en plus active sur ces sujets (Hjalager, 2010).

Pour conclure cette introduction, nous souhaitons remercier RFI Tourisme, une initiative du Conseil régional des Pays de Loire, grâce à laquelle ce travail a pu être mené.

\section{BIBLIOGRAPHIE}

Olivier BESSY et André SUCHET, « Une approche théorique de l'événementiel sportif », Mondes du tourisme, $n^{\circ} 11,2015$.

Laurent BоттІ, Pour une gestion de la touristicité des territoires, Baixas : Balzac Éditeur, 2011.

Marc BOYER, L'invention du tourisme. Paris : Gallimard,1996.

Marc BOYER et Philippe VIALLON (1994), La communication touristique, Paris : PUF.

Pierre BOURDIEU, La distinction. Paris : Éditions de Minuit, 1979.

Vincent COËFFÉ et Philippe VIOLIER, « Les lieux du tourisme : de quel(s) paradis parle-t-on? Variations sur le thème de l'urbanité touristique », Articulo - Journal of Urban Research, nº 4, 2008 [http://articulo.revues.org/158; DOI : 10.4000/articulo.158 ].

John H. FALK et Lynn D. DIERKING, Learning from Museums. Visitors experiences and the making of meaning, Wallnut creek : Alta Mira Press, 2000.

Christophe GUIBERT, «Introduction au dossier Sport, tourisme et production de territoires », Mondes du tourisme, $\mathrm{n}^{\circ}$ 11, 2011.

Anne-Mette HJALAGER, «A review of innovation research in tourism », Tourism Management, $\mathrm{n}^{\circ} 31$, 2010.

Marc LOHEZ, «Thermalisme et tourisme : les évolutions récentes ", L'information géographique, vol. $64, \mathrm{n}^{\circ} 4,2000$.

OMT, Faits saillants, 2017.

Marielle SALVADOR et Alain JOLIBERT, "Cahier : L'innovation dans le tourisme, l'heure des défis », Management \& Avenir, $\mathrm{n}^{\circ}$ 91, 2017.

Thorsten VEBLEN, Théorie de la classe de loisirs, Paris : Gallimard, 1979.

\section{NOTES}

1. C'est ainsi qu'était caractérisée la haute montagne avant qu'elle ne devienne ludique grâce à l'alpinisme, au ski ou à la randonnée.

2. Source : OMT, Faits saillants, 2017. 
3. Ibidem.

4. Voir : https://www.entreprises.gouv.fr/services/innovation-servicielle

\section{RÉSUMÉS}

L'innovation est à l'oeuvre dans de nombreux domaines, notamment dans le tourisme. Fortement liée à l'invention et à la nouveauté, elle est considérée comme positive, car elle accroit les possibilités de choix pour le touriste et développe l'activité économique. Elle est aussi très polymorphe et cela explique la difficulté à la définir. On peut néanmoins affirmer qu'elle est principalement liée aux nouvelles technologies, centrée sur l'usager et qu'elle se situe surtout dans le domaine des loisirs.

Innovation is apparent in many branches of industry, including tourism. Innovation is seen as a key composent to the success of the economic activity. It ingrates choices for the tourists. It is considered as positive, it signifies novelty even of invention. there's no consensus on the definition of innovation because takes many forms. however, it is primarily driven by new technologies and it is often focused on the user. Many innovations are in the field of the leisure activities.

\section{AUTEURS}

\section{AUDE DUCROQUET}

Université d'Angers

\section{PHILIPPE VIALLON}

Université de Strasbourg,

Chaire Unesco « Pratiques journalistiques et médiatiques » 